\title{
The Potential Use of a Ketogenic Diet in Pancreatobiliary Cancer Patients After Pancreatectomy
}

\author{
JU HYE OK ${ }^{1}$, HOSUN LEE ${ }^{1}$, HAE-YUN CHUNG ${ }^{2}$, SUNG HWAN LEE ${ }^{3,4}$, \\ EUN JEONG CHOI ${ }^{5}, \mathrm{CHANG}^{\mathrm{MOO}} \mathrm{KANG}^{3,4}$ and SONG MI LEE ${ }^{*}$ \\ ${ }^{1}$ Department of Nutrition Care, Severance Hospital, Yonsei University Health System, Seoul, Republic of Korea; \\ ${ }^{2}$ Department of Food and Nutrition, Soongeui Women's University, Seoul, Republic of Korea; \\ ${ }^{3}$ Division of Hepatobiliary and Pancreatic Surgery, Department of Surgery, \\ Yonsei University College of Medicine, Seoul, Republic of Korea; \\ ${ }^{4}$ Pancreatobiliary Cancer Center, Yonsei Cancer Center, Severance Hospital, Seoul, Republic of Korea; \\ ${ }^{5}$ Department of Food and Nutrition, Hanyang Women's University, Seoul, Republic of Korea
}

\begin{abstract}
Background/Aim: High-carbohydrate diets are generally provided to post-pancreatectomy cancer patients. Low energy density of this diet may obstruct proper energy intake and recovery. This study aimed to assess the effects of high-fat, high-energy ketogenic diet $(K D)$ in these patients. Patients and Methods: After pancreatectomy, 9 patients were provided with general diet $(G D)$ while 10 were served $K D$. Meal compliance, energy intake rate, meal satisfaction and presence of complications were monitored throughout hospital stay. Data on nutritional status, serum lipids and body composition were collected and compared between groups. Results: Meal compliance, energy intake rate and meal satisfaction score were higher in KD. There were no differences in complications, nutritional status and serum lipids. The decrease in body cell mass (BCM) was greater in GD. Conclusion: Postpancreatectomy cancer patients who consumed KD had a higher energy intake and BCM. These results suggest the potential use of $K D$ as an adjuvant anti-cancer therapy.
\end{abstract}

Pancreatic cancer accounts for $2.4 \%$ of the cancer incidence worldwide and has a particularly poor prognosis, resulting in

This article is freely accessible online.

Correspondence to: Song Mi Lee, Ph.D., Department of Nutrition Care, Severance Hospital, Yonsei University Health System, 50-1 Yonsei-Ro, Seodaemun-Gu, Seoul 03722, Republic of Korea. Tel: +82 222286959, e-mail: nutrpine@yuhs.ac and Chang Moo Kang, M.D., Ph.D., Division of Hepatobiliary and Pancreatic Surgery, Department of Surgery, Yonsei University College of Medicine, 501 Yonsei-Ro, Seodaemun-Gu, Seoul 03722, Republic of Korea. Tel: +82 222282135, e-mail: cmkang@yuhs.ac

Key Words: Pancreatic cancer, pancreatectomy, ketogenic diet, adjuvant anti-cancer therapy.
$4.0 \%$ mortality. Gallbladder cancer accounts for $1.3 \%$ of all cancers with a mortality of $1.7 \%$ (1). Pancreatic cancer in South Korea was the eighth most common at $3 \%$ and gallbladder and other biliary cancers were the ninth most common at $2.9 \%$ in 2015 which, with regard to mortality, were ranked fifth with a rate of $7.2 \%$ and sixth with a rate of $5.6 \%$, respectively. The five-year relative survival rate of pancreatic cancer was the lowest of all cancers at $10.8 \%$ (2).

Radical resection is the only method that can completely cure pancreatobiliary cancer, as complete tumor removal can considerably reduce the risk of cancer recurrence and increase patient survival rates $(3,4)$. However, patients who undergo pancreatectomy are more susceptible to malnourishment and weight loss due to complications such as pancreatic fistula, delayed gastric emptying, dumping syndrome, impaired digestive enzyme secretion, dysfunctional nutrient absorption, diarrhea, and steatorrhea (5). Furthermore, delayed or reduced food intake after surgery due to difficulty in consuming food can also induce weight loss. This weight loss may subsequently impact cancer cachexia caused by malignant tumors, malnutrition, and reduced quality of life $(6,7)$. Multiple studies have reported that cancer patients experience at least $10 \%$ of their total weight loss within six months after surgery due to cancer cachexia and that death caused by serious weight loss accounts for $80 \%$ of deaths caused by pancreatic cancer (8). According to a 2015 Korean study, $60 \%$ of patients who undergo pancreatoduodenectomy experience at least two factors that hinder food intake and subsequent weight loss, suggesting the need to improve the standard meals provided after pancreatectomy (9).

Recently, ketogenic diets (KD) have gained traction as a potential adjuvant anticancer therapy for targeting specific metabolic reactions of cancer tissues (10). Although its exact metabolic mechanisms are unknown, it has been speculated that ketosis caused by fasting inhibits cancer cell growth and 
that the restricted use of glucose during KD increases oxidative stress in cancer cells (11). Several clinical trials investigating the effects of KD in various cancer patients are currently being conducted. There have been relatively few studies on pancreatobiliary cancer patients, but these patients are customarily prescribed a high-carbohydrate, low-fat diet after pancreatectomy due to supposedly poor nutrient absorption, fat in particular. However, high-carbohydrate diets result in approximately $30 \%$ of required energy intake due to their low-calorie densities, making it difficult for patients to satisfy nutritional requirements after surgery. On the other hand, KD is high in fat and low in carbohydrates, which means that a patient can consume a sufficient amount of energy by consuming only a relatively small amount of food.

Therefore, this study aimed to investigate whether a high-fat, low-carbohydrate KD provided after radical pancreatectomy to treat pancreatobiliary cancer can improve dietary intake and patients' satisfaction compared to the general high-carbohydrate diet. Further, this study also investigated whether KD can improve patients' nutritional status and cancer-related clinical parameters such as serum lipids and lean body mass.

\section{Materials and Methods}

Ethics statement. This study was approved by the Institutional Review Board at Severance Hospital, Seoul, Korea (4-2016-079).

Study design. This prospective controlled intervention study was conducted from November 2016 to May 2017. Participants were adults who were diagnosed with a pancreatobiliary cancer, defined as either pancreatic cancer, duodenal cancer, distal biliary tract cancer, or carcinoma of the ampulla of vater, and were hospitalized to undergo pancreaticoduodenectomy or distal pancreatectomy. The patients who were excluded were pregnant women, severe diabetes patients with diabetic complications, hyperlipidemia patients with cardiovascular complications, patients with renal impairment below $90 \%$ of normal glomerular filtration rate, and illiterate patients. All participants understood the purpose of the study and voluntarily consented to participate in the study. Thirty patients were enrolled during the study period and sequentially assigned to either general diet (GD) group or KD group. After excluding 11 dropouts, a total of 19 patients were included in the data analysis (Figure 1).

Treatment. Anthropometric measurements, nutritional evaluation, and body composition analysis were performed before surgery (preOP), before discharge (DC), and at the first outpatient follow-up day (OPD) for all patients and general information (e.g., sex, age, diagnosis, type of surgery), blood and urine test results, and other relevant data were collected from electronic medical records (Figure 2).

After consuming a carbohydrate-supplement drink on the first day after surgery, the patients gradually began taking GD or KD in full liquid form and soft diet from postoperative day 3 . The patients were instructed to record the percentage of each meal that they consumed in a daily $\log$ from the first day of the full liquid diet until the day of discharge. A research dietitian periodically visited patient to monitor the record keeping and check for problems with meal intake. Meal compliance was defined as the average

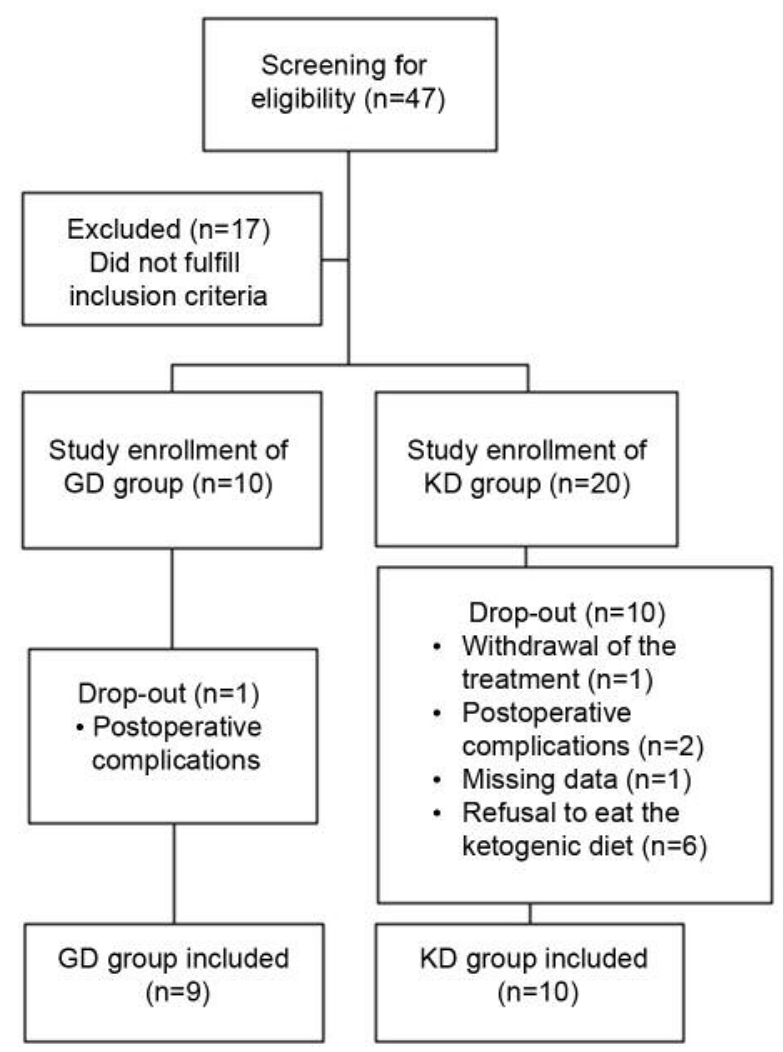

Figure 1. Flow chart of study population. GD: General diet; KD: ketogenic diet.

percentage of provided meals consumed. At discharge, patients in KD group were given instructions on ketogenic diet and encouraged to consume KD at home. At OPD, the patients' 3-day food diaries (two weekdays and one weekend day) were examined during an interview. Dietary intakes were analyzed using the CAN-Pro 4.0 (Computer-Aided Nutritional Program) developed by Korean Nutrition Society to compute daily energy and protein intakes. Energy and protein intake rates were then calculated based on the energy and protein requirements of each patient. Energy requirement was calculated using the Mifflin-St. Jeor equation (12) with an estimated resting metabolic rate and a $25 \%$ activity factor. Protein requirement was set at $1 \mathrm{~g} / \mathrm{kg}$ body weight.

Nutritional assessments were performed using the patientgenerated subjective global assessment (PG-SGA), which is generally used for nutritional assessments of cancer patients (13). Body composition was analyzed using BIA (Biospace, Seoul, Korea) and generated body cell mass (BCM), body fat mass (BFM), and skeletal muscle mass (SMM) data. Serum cholesterol, HDLcholesterol, LDL-cholesterol, triglyceride, C-reactive protein (CRP), and urine ketone were collected from electronic medical records during study period. Postoperative complications were investigated using the Clavien-Dindo classification. Patients with grade III or higher complications were defined as having developed complications for the purposes of this study (14).

General diet vs. ketogenic diet. Westman et al. (15) found that ketones are detected in the urine of people who consume 20-50 g 


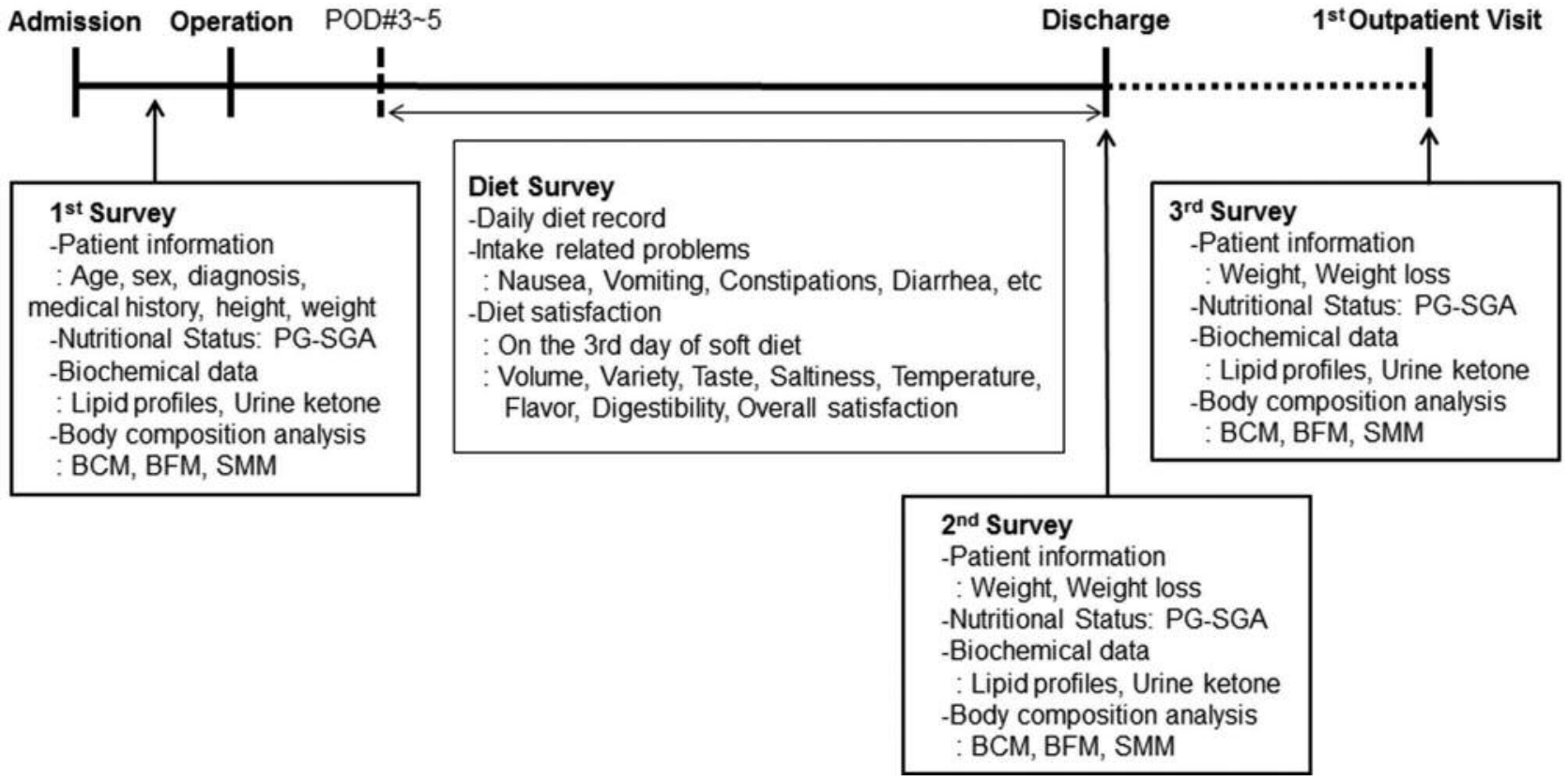

Figure 2. Timing of survey during the study. CLD: Clear liquid diet; FLD: full liquid diet; SD: soft diet; PG-SGA: patient generated-subjective global assessment; POD: postoperative day; BCM: body cell mass; BFM: body fat mass; SMM: skeletal muscle mass.

of carbohydrates per day, so the subjects in KD group were provided with $3-6 \%$ of their daily energy as carbohydrates to induce ketosis. To promote postoperative recovery and prevent loss of body proteins, $1 \mathrm{~g} / \mathrm{kg}$ of high-quality protein was provided. Further, 70$80 \%$ of energy was provided through fats to reach a ketogenic ratio of 1.05-1.75:1 (Fat: Carbohydrate+Protein, w:w). Fats were provided mainly in the form of vegetable oil, which was rich in unsaturated fat, and nuts, which had high levels of monounsaturated fats and phytosterols. Animal fats, found in products such as whipped cream and butter, were only used in small amounts. The general diet of this study was similar to usual Korean diet with carbohydrate:protein:fat (C:P:F) ratio of 55-65:7-20:15-30 (Table I).

Data analysis. Results are expressed as geometric means \pm standard deviation (SD). Data were verified for normality (Shapiro-Wilk test) and, when necessary, were log-transformed for normal distribution before further statistical analysis. Differences in general characteristics, meal compliance, energy and protein intake rates, meal satisfaction score, presence of meal intake-related problems, PGSGA grade and urine ketone detection between GD and KD groups were compared by the Student's $t$ test or Fisher's exact test. To determine whether there was a group $\mathrm{x}$ day interaction in meal compliance, dietary intake, serum lipid levels and body composition variables, 2-way ANOVA was performed. The changes of mean serum lipid levels and body composition variables over time in each group were analyzed by a repeated-measures 1-way ANOVA, followed by the Tukey-Kramer test for multiple comparisons. A Student's $t$ test was performed to compare the difference in changes of serum lipid levels and body composition variables among groups (OPD - preOP). For this analysis, baseline-adjusted values were used. A $p$-value $<0.05$ was considered significant. Statistical analyses were performed using SAS version 9.4 (SAS Inc., Cary, NC, USA)

\section{Results}

Subject characteristics. Of the 10 participants assigned to the GD group, 1 was withdrawn from the study due to postoperative complications. Of the 20 participants assigned to the KD group, 10 were withdrawn from the study for stopping treatment $(n=1)$, postoperative complications $(n=2)$, insufficient data $(n=1)$, and refusal to comply with the study meal $(n=6)$. As a result, 9 participants in the GD group and 10 participants in the KD group completed the study. Table II shows a summary of their general information and anthropometric characteristics. There were no significant differences in the sex ratio, nutritional status, diagnosis, comorbidity, and type of surgery between the two groups. The GD group was older on average and had a lower average BMI than the KD group, but these differences were not statistically significant.

Meal compliance and rate of energy and protein intake. The first day during which participants began full liquid diets was designated as day 1. Daily meal compliance and energy and protein intake rates from day 1-10 were analyzed. The average meal compliance rate and energy intake rate in KD group were significantly higher than the GD group (Table III). Protein intake rate was also higher in KD group, but the difference was not statistically significant $(p=0.0710)$.

Student's $t$-test was performed to compare the two groups at 10 points over the data collection period. The KD group had significantly higher meal compliance rates than the GD group 
Table I. Composition of General and Ketogenic diets.

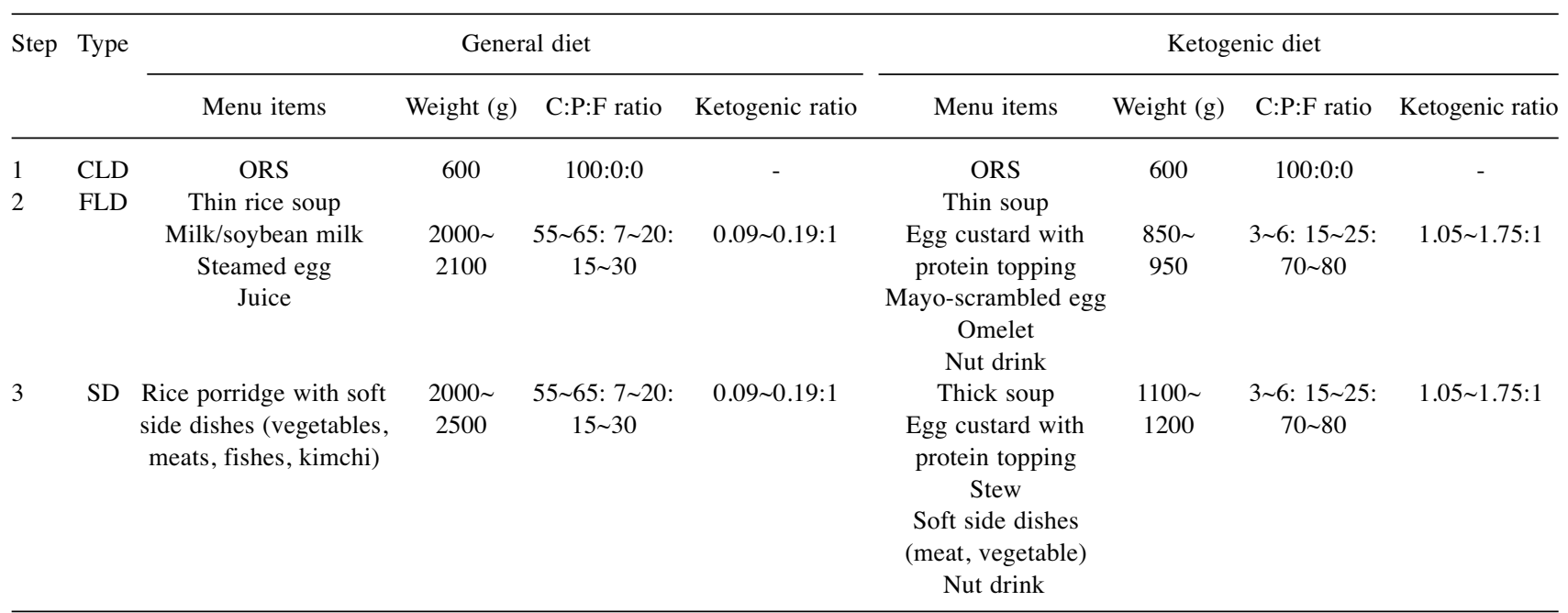

CLD: Clear liquid diet; FLD: full liquid diet; SD: Soft Diet; C:P:F ratio: Carbohydrate:Protein:Fat ratio; ORS: oral rehydration solution (Carbohydrate 25 g/200 ml/can, Daesang Wellife Co., Seoul, Korea).

during days $1-5(p<0.05)$. Energy and protein intake rates were also higher in KD group at day $1(p<0.01$, Figure 3$)$.

Dietary intake was also analyzed from DC to OPD based on the food records of the patients. Even though the KD group consumed slightly more fat and less carbohydrate, the dietary $\mathrm{C}: \mathrm{P}: \mathrm{F}$ ratio of the two groups did not differ. There were also no statistically significant differences in the energy and protein intake rates between the two groups (data now shown).

Meal satisfaction score and meal intake-related problems. On the third day of the soft diet, participants were surveyed about their meal satisfaction using a self-administered questionnaire (Table IV). There were no differences in satisfaction with the taste, saltiness, and smell of the meals between the two groups, but the KD group had higher volume, temperature, digestibility, and overall satisfaction scores than the GD group $(p<0.05)$.

A research dietitian periodically visited patients to check for problems with dietary intake such as nausea, vomiting, constipation and diarrhea. There was no difference in proportion of patients suffering from meal intake-related problems. The total number of complaints were higher in GD group, but the difference was not statistically significant (Table V).

PG-SGA score. Figure 4 shows a comparison of the changes in patients' nutritional status during the study period. Nutritional states were classified into three grades according to the PG-SGA score: good (SGA A) or poor and requiring intervention (SGA B, SGA C). The proportion of patients in each grade was not different among groups at preOP. The percentage of SGA B and SGA C patients in both groups increased at DC, but there was no significant difference in these rates. Even though more patients in GD group were in either SGA B or SGA C grade at DC, but the difference from KD was not statistically significant.

Biochemical indices. Table VI shows the biochemical test results from throughout the study period. The KD group had a significantly lower cholesterol, HDL and LDL levels at DC than preOP $(p<0.01)$. The GD group's HDL level decreased significantly, also $(p<0.001)$. By OPD, these reduced levels returned to those comparable to preOP. The amount that this level was reduced (DC-preOP, OPD-preOP) was not significantly different between the two groups. The CRP level was significantly higher at DC in GD and KD groups ( $p<0.01$ and $p<0.001$, respectively). At OPD, CRP level was not significantly different from preOP level in GD group. In $\mathrm{KD}$, CRP level was still higher than that of preOP. However, the amount that this level was changed was not significantly different between groups, possibly due to large variation.

There were no group $\mathrm{x}$ day interactions in levels of cholesterol, HDL, LDL, TG and CRP.

Body composition. Table VII shows the body composition analysis results from throughout the study period.

When compared to preOP levels, the body weight, BCM, and SMM of both groups were significantly lower at OPD $(p<0.001)$. And the decreases in BCM were significantly greater in GD group $(p<0.05)$. SMM decrease was also greater in GD group, though not significant $(p=0.054)$. While BFM of the GD group was similar or slightly higher 
Table II. Baseline characteristics of subjects ${ }^{1}$.

\begin{tabular}{|c|c|c|c|}
\hline Variable & GD $(n=9)$ & $\mathrm{KD}(\mathrm{n}=10)$ & $p$-Value \\
\hline Age (yr) & $\begin{array}{c}66.3 \pm 9.8 \\
(54-78)\end{array}$ & $\begin{array}{c}57.8 \pm 7.3 \\
(49-70)\end{array}$ & 0.053 \\
\hline Gender, n (\%) & & & 1.000 \\
\hline M & $6(66.7)$ & $6(60.0)$ & \\
\hline $\mathrm{F}$ & $3(33.3)$ & $4(40.0)$ & \\
\hline BMI $\left(\mathrm{kg} / \mathrm{m}^{2}\right)$ & $22.2 \pm 2.6$ & $24.0 \pm 2.2$ & 0.079 \\
\hline PG-SGA Score & $4.7 \pm 5.7$ & $3.4 \pm 2.8$ & 0.720 \\
\hline Diabetes mellitus, n (\%) & & & 1.000 \\
\hline No & $8(8.9)$ & $9(90.0)$ & \\
\hline Yes & $1(11.1)$ & $1(10.0)$ & \\
\hline Hypertension, n (\%) & & & 0.350 \\
\hline No & $7(77.8)$ & $5(50.0)$ & \\
\hline Yes & $2(22.2)$ & $5(50.0)$ & \\
\hline Diagnosis, n (\%) & & & 0.805 \\
\hline Ampulla of vater cancer & $2(22.2)$ & $1(10.0)$ & \\
\hline Common bile duct cancer & $3(33.3)$ & $2(20.0)$ & \\
\hline Cholangiocarcinoma & $2(22.2)$ & $1(10.0)$ & \\
\hline Duodenal cancer & $0(0.0)$ & $1(10.0)$ & \\
\hline Pancreatic cancer & $2(22.2)$ & $4(40.0)$ & \\
\hline Neuroendocrine Tumor & $0(0.0)$ & $1(10.0)$ & \\
\hline Cancer stage, n (\%) & & & 0.534 \\
\hline I & $1(11.1)$ & $3(30.0)$ & \\
\hline II & $1(11.1)$ & $2(20.0)$ & \\
\hline III & $6(66.7)$ & $3(30.0)$ & \\
\hline IV & $1(11.1)$ & $2(20.0)$ & \\
\hline Operation name, n (\%) & & & 1.000 \\
\hline DP & $2(22.2)$ & $2(20.0)$ & \\
\hline PPPD & $7(77.8)$ & $8(80.0)$ & \\
\hline Operation type, n (\%) & & & 1.000 \\
\hline Laparoscopic surgery & $7(77.8)$ & $8(80.0)$ & \\
\hline Open surgery & $2(22.2)$ & $2(20.0)$ & \\
\hline LOS (day) & $15.8 \pm 11.2$ & $12.0 \pm 5.8$ & 0.604 \\
\hline
\end{tabular}

${ }^{1}$ Means \pm SDs for continuous variables and $\mathrm{N}(\%)$ for categorical variables. The difference among groups was tested by the Student's $t$ test or Fisher's exact test. None of the variables was not significantly different between groups at $p<0.05$. BMI: Body mass index; PG-SGA: patient-generated subjective global assessment; DP: distal pancreatectomy; PPPD: pylorus-preserving pancreaticoduodenectomy; LOS: length of stay.

Table III. Meal compliance, energy and protein intake rates among GD and KD groups during 10 days after the start of the meal ${ }^{1}$.

\begin{tabular}{lcc}
\hline & $\begin{array}{c}\text { GD group } \\
(\mathrm{n}=9)\end{array}$ & $\begin{array}{c}\text { KD group } \\
(\mathrm{n}=10)\end{array}$ \\
\hline $\begin{array}{l}\text { Average meal compliance }(\%)^{2 * * *} \\
\text { Average intake rate }(\%)\end{array}$ & $33.9 \pm 16.6$ & $69.1 \pm 19.6$ \\
$\begin{array}{l}\text { Energy* } \\
\text { Protein }\end{array}$ & $38.5 \pm 21.9$ & $61.3 \pm 19.0$ \\
\hline
\end{tabular}

${ }^{1}$ Means \pm SDs; ${ }^{2}$ Significantly different between groups (Student's $t$-test): $* p<0.05, * * p<0.01, * * * p<0.001$.
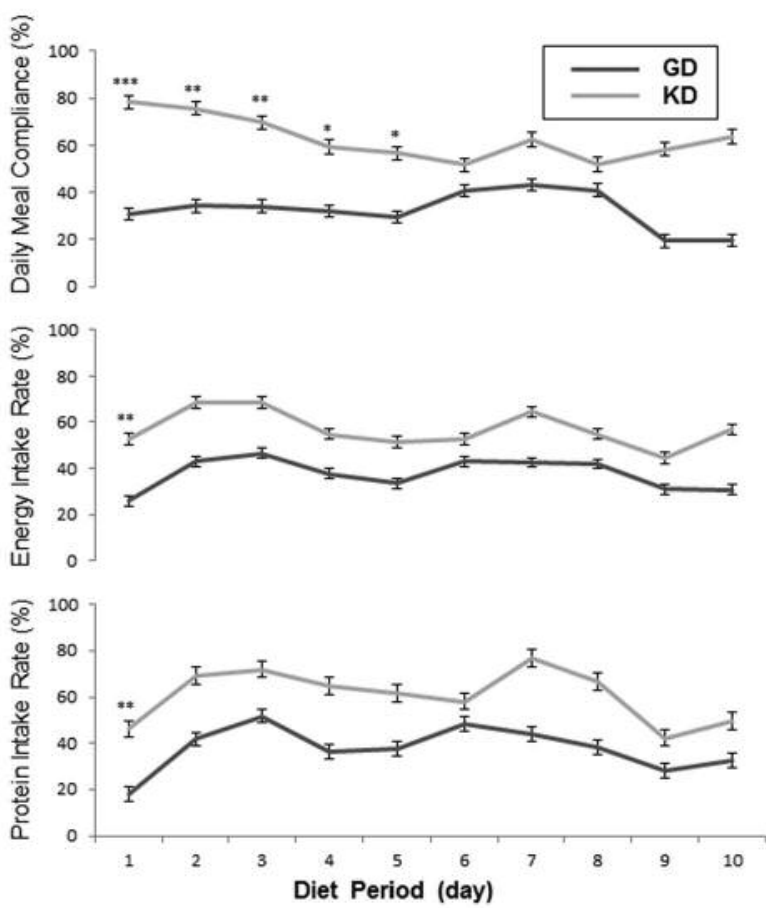

Figure 3. Daily meal compliance, energy and protein intake rates during the diet period. Values are presented as means $\pm S D$ s. Significantly different between groups at each time point (Student's t-test): ${ }^{*} p<0.05$, $* * p<0.01, \quad * * * p<0.001$. Energy Intake Rate: (actual energy intake)/(recommended energy intake) $\times 100$; Protein Intake Rate: (actual protein intake)/(recommended protein intake $) \times 100$.

after surgery than before surgery, that of KD group was significantly lower at OPD than preOP $(p<0.05)$. The degree of the changes in BFM was smaller in KD group, though the difference was not significant $(p=0.086)$.

Urine ketones. Table VIII shows the urine ketone detection before surgery, at discharge, and at outpatient visit. Before surgery, urine ketone bodies were detected in one patient in the KD group and none of the patients in the GD group. At DC, $70.0 \%$ of patients in the KD group had ketone bodies in their urine while only $22.2 \%$ of patients in the GD group did, but this difference was not statistically significant $(p=0.069)$. There was no statistically significant difference between the two groups in the number of patients with urine ketone bodies at OPD.

Other than the three points mentioned above, numerous urine tests were performed on the patients during hospital stay. We pooled all the test results and calculated the ratio of times that each patient had urine ketone bodies. The ketone detection frequency was $22.2 \pm 23.7 \%$ in the GD group and $50.8 \pm 35.1 \%$ in the KD group, but the difference between the two groups was not statistically significant ( $p=0.065$, data not shown). 
Table IV. Comparison of meal satisfaction scores between GD and KD groups $^{1}$.

\begin{tabular}{lcc}
\hline Variables & $\begin{array}{c}\text { GD group } \\
(\mathrm{n}=9)\end{array}$ & $\begin{array}{c}\text { KD group } \\
(\mathrm{n}=10)\end{array}$ \\
\hline Meal Volume $2 * * *$ & $2.2 \pm 1.3$ & $7.3 \pm 2.1$ \\
Composition of menu & $3.0 \pm 1.2$ & $4.8 \pm 2.7$ \\
Taste & $4.2 \pm 2.5$ & $4.1 \pm 1.7$ \\
Salinity & $4.7 \pm 2.4$ & $5.9 \pm 2.5$ \\
Temperature* & $5.5 \pm 2.2$ & $8.3 \pm 2.4$ \\
Smell & $4.1 \pm 1.2$ & $5.2 \pm 2.1$ \\
Digestibility*** & $2.8 \pm 1.1$ & $7.1 \pm 2.6$ \\
Overall satisfaction** & $3.8 \pm 1.1$ & $6.2 \pm 1.8$ \\
\hline
\end{tabular}

${ }^{1}$ Means \pm SDs; ${ }^{2}$ Significantly different between groups (Student's $t$-test): $* p<0.05, * * p<0.01, * * * p<0.001$.

Table V. Frequency of meal intake-related problems ${ }^{l}$.

\begin{tabular}{lcc}
\hline & $\begin{array}{c}\text { GD group } \\
(\mathrm{n}=9)\end{array}$ & $\begin{array}{c}\text { KD group } \\
(\mathrm{n}=10)\end{array}$ \\
\hline $\begin{array}{l}\text { Anorexia, } \mathrm{n}(\%) \\
\text { positive }\end{array}$ & $8(88.9)$ & $5(50.0)$ \\
negative & $1(11.1)$ & $5(50.0)$ \\
Nausea, $\mathrm{n}(\%)$ & & \\
positive & $4(44.4)$ & $3(30.0)$ \\
negative & $5(55.6)$ & $7(70.0)$ \\
$\begin{array}{l}\text { Vomiting, } \mathrm{n}(\%) \\
\text { positive } \\
\text { negative }\end{array}$ & $3(33.3)$ & $1(10.0)$ \\
Constipation, $\mathrm{n}(\%)$ & $6(66.7)$ & $9(90.0)$ \\
positive & & \\
negative & $1(11.1)$ & $0(0.0)$ \\
Abdomen Pain, $\mathrm{n}(\%)$ & $8(88.9)$ & $10(100.0)$ \\
positive \\
negative
\end{tabular}

${ }^{1}$ The difference in proportion among groups was tested by Fisher's exact test. None of the variables was not significantly different between groups at $p<0.05$.

Complications after surgery. The incidence of postoperative complications was compared using the Clavien-Dindo classification (14). Whereas none of the patients in the KD group developed a postoperative complication, two patients $(22.2 \%)$ in the GD group developed complications. However, this difference was not statistically significant (data not shown).

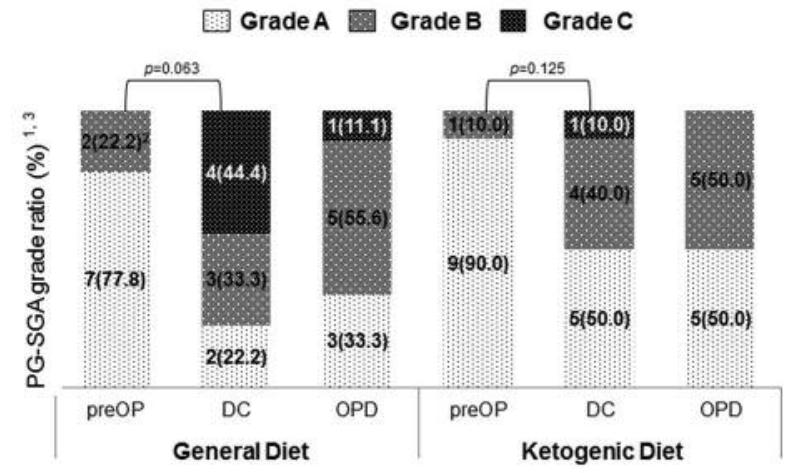

Figure 4. Change of PG-SGA grade among groups. ${ }^{1} P G-S G A$ : Patientgenerated subjective global assessment; ${ }^{2} n(\%) ;{ }^{3}$ The differences among groups at each time point were tested by the Fisher's exact test. There was no significant difference between groups at $p<0.05$. Changes from preOP to DC in each group were tested by McNemar test. None of the changes was not statistically significant. preOP: Measurements of day before surgery; DC: measurements of day before discharge; OPD: measurements of first outpatient visits.

Table VI. Changes of biochemical indices during study period ${ }^{l}$.

\begin{tabular}{lccc}
\hline Variables & $\begin{array}{c}\text { GD group } \\
(\mathrm{n}=9)\end{array}$ & $\begin{array}{c}\text { KD group } \\
(\mathrm{n}=10)\end{array}$ & $p$-Value \\
& & & \\
\hline Cholesterol $(\mathrm{mg} / \mathrm{dl})$ & & & \\
preOP & $155.7 \pm 46.1$ & $180.2 \pm 45.7^{\mathrm{a}}$ & - \\
DC & $136.6 \pm 30.3$ & $145.2 \pm 20.2^{\mathrm{b}}$ & \\
OPD & $156.0 \pm 48.4$ & $173.8 \pm 32.1^{\mathrm{a}}$ & \\
HDL-C $(\mathrm{mg} / \mathrm{dl})$ & & & \\
preOP & $43.8 \pm 11.2^{\mathrm{a}}$ & $43.4 \pm 9.7^{\mathrm{a}}$ & - \\
DC & $28.6 \pm 9.7^{\mathrm{b}}$ & $25.2 \pm 3.9^{\mathrm{b}}$ & \\
OPD & $42.4 \pm 6.9^{\mathrm{a}}$ & $38.4 \pm 8.8^{\mathrm{a}}$ & \\
LDL-C (mg/dl) & & & \\
preOP & $86.0 \pm 36.5$ & $110.8 \pm 37.0^{\mathrm{a}}$ & - \\
DC & $81.3 \pm 29.3$ & $88.3 \pm 17.8^{\mathrm{b}}$ & \\
OPD & $97.4 \pm 41.9$ & $108.4 \pm 28.3^{\mathrm{a}}$ & \\
TG (mg/dl) & & & \\
preOP & $120.7 \pm 74.5$ & $131.8 \pm 86.1$ & - \\
DC & $130.4 \pm 67.2$ & $175.8 \pm 108.5$ & \\
OPD & $110.5 \pm 51.1$ & $125.7 \pm 49.8$ & \\
CRP $(\mathrm{mg} / \mathrm{l})$ & & & \\
preOP & $14.1 \pm 35.0$ & $2.7 \pm 3.7$ & - \\
DC & $38.9 \pm 26.1$ & $85.6 \pm 62.1$ & \\
OPD & $10.0 \pm 11.8$ & $25.6 \pm 36.6$ & \\
\hline
\end{tabular}

${ }^{1}$ Means \pm SDs. Values in a column with different superscript letters are significantly different, $p<0.05$ (repeated-measures one-factor ANOVA followed by a Tukey-Kramer test). For this analysis, baseline-adjusted values were used; ${ }^{2}$ The differences between groups in changes from preOP to OPD were tested by the Student's $t$-test. None of the variables were significantly different between groups at $p<0.05$. HDL-C: Highdensity lipoprotein cholesterol; LDL-C: low-density lipoprotein cholesterol; TG: triglyceride, CRP: C-reactive protein; preOP: measurements of day before surgery; DC: measurements of day before discharge; OPD: measurements of first outpatient visit. 
Table VII. Changes of body composition during study period ${ }^{l}$.

\begin{tabular}{lccc}
\hline Variables & $\begin{array}{c}\text { GD group } \\
(\mathrm{n}=8)\end{array}$ & $\begin{array}{c}\text { KD group } \\
(\mathrm{n}=9)\end{array}$ & $p$-Value ${ }^{2}$ \\
\hline BW (kg) & & & \\
preOP & $56.2 \pm 7.2^{\mathrm{a}}$ & $64.6 \pm 11.2^{\mathrm{a}}$ & 0.475 \\
DC & $54.0 \pm 6.7^{\mathrm{b}}$ & $61.2 \pm 9.5^{\mathrm{b}}$ & \\
OPD & $52.7 \pm 7.2^{\mathrm{b}}$ & $60.6 \pm 9.5^{\mathrm{b}}$ & \\
BCM $(\mathrm{kg})$ & & & \\
preOP & $27.4 \pm 4.7^{\mathrm{a}}$ & $28.9 \pm 4.5^{\mathrm{a}}$ & 0.049 \\
DC & $25.7 \pm 4.6^{\mathrm{b}}$ & $27.3 \pm 5.0^{\mathrm{b}}$ & \\
OPD & $24.5 \pm 4.2^{\mathrm{c}}$ & $27.0 \pm 5.0^{\mathrm{b}}$ & \\
BFM $(\mathrm{kg})$ & & & \\
preOP & $13.7 \pm 6.2^{2}$ & $18.2 \pm 5.2^{\mathrm{a}}$ & 0.086 \\
DC & $14.0 \pm 5.8$ & $17.6 \pm 5.1^{\mathrm{b}}$ & \\
OPD & $14.2 \pm 6.4$ & $17.1 \pm 4.9^{\mathrm{b}}$ & \\
SMM $(\mathrm{kg})$ & & & \\
preOP & $23.0 \pm 4.3^{\mathrm{a}}$ & $24.3 \pm 4.1^{\mathrm{a}}$ & 0.054 \\
DC & $21.4 \pm 4.2^{\mathrm{b}}$ & $22.8 \pm 4.6^{\mathrm{b}}$ & \\
OPD & $20.2 \pm 3.8^{\mathrm{c}}$ & $22.6 \pm 4.5^{\mathrm{b}}$ & \\
\hline
\end{tabular}

${ }^{1}$ Means \pm SDs. Values in a column with different superscript letters are significantly different, $p<0.05$ (repeated-measures one-factor ANOVA followed by a Tukey-Kramer test). For this analysis, baseline-adjusted values were used. ${ }^{2}$ The differences between groups in changes from preOP to OPD were tested by Student's $t$-test. BW: Body weight; BCM: body cell mass; BFM: body fat mass; SMM: skeletal muscle mass; preOP: measurements of day before surgery; DC: measurements of day before discharge; OPD: measurements of first outpatient visits.

\section{Discussion}

Schiesser et al. (16) reported that patients who underwent digestive system surgeries ate less per sitting and overall due to indigestion, early satiety while eating, abdominal fullness, and psychological fear of eating. Therefore, this study aimed to help pancreatobiliary cancer patients improve nutrient intake and recovery after surgery by providing a high-fat, high-calorie KD.

The KD used in this study was developed such that patients were able to obtain a large amount of energy from more frequent, smaller meals given the difficulty that such patients typically have with eating after surgery. In this study diet, patients were provided three meals and three snacks. Whereas the meals that the GD group received were $\sim 750 \mathrm{~g}$, the meals that the KD group received were $\sim 210 \mathrm{~g}$ and snacks were $\sim 160 \mathrm{~g}$. A large portion of the KD was made up of nuts and oils, giving the KD 1.5 times more calories/g and 2 times more protein/g than the GD.

The findings of this study showed that postoperative patients who received the KD had greater meal compliance than patients who received the GD. In addition, patients in the KD group had higher energy intake rates, which is similar to the findings of Wallengren et al. (17) who found that increasing the nutritional density of meals received by cancer
Table VIII. Comparison of urine ketone detection among GD and KD groups $^{1}$

\begin{tabular}{lll}
\hline & $\begin{array}{c}\text { GD group } \\
(\mathrm{n}=9)\end{array}$ & $\begin{array}{c}\text { KD group } \\
(\mathrm{n}=10)\end{array}$ \\
\hline $\begin{array}{l}\text { preOP } \\
\text { Urine ketone, } \mathrm{n}(\%)\end{array}$ & \\
$\quad$ Negative & $9(100.0)$ & $9(90.0)$ \\
$\quad$ Positive & $0(0.0)$ & $1(10.0)$ \\
DC & & \\
$\quad$ Urine ketone, $\mathrm{n}(\%)$ & $7(77.8)$ & $3(30.0)$ \\
$\quad$ Negative & $2(22.2)$ & $7(70.0)$ \\
Positive & & \\
OPD & & \\
$\quad$ Urine ketone, $\mathrm{n}(\%)$ & $7(77.8)$ & $5(50.0)$ \\
Negative & $2(22.2)$ & $5(50.0)$ \\
Positive & & \\
\hline
\end{tabular}

${ }^{1}$ The differences among groups at each time point were tested by Fisher's exact test. There was no significant difference between groups at $p<0.05$. preOP: Measurements of day before surgery; DC: measurements of day before discharge; OPD: measurements of first outpatient visits.

patients increased their energy intake rates. Amelioration of nutritional status through proper nutritional intake is important because it improves quality of life and survival in cancer patients undergoing chemotherapy following surgery (18-19). In this study, six patients assigned to KD refused to consume the diet. Of the six, three patients said they did not like KD because it is different from usual Korean diet which is low in fat and high in carbohydrate. The other three patients complained about the greasy smell from butter and cream of $\mathrm{KD}$. The mean length of hospital stays for KD subjects who completed the study was $12 \pm 5.8$ days while it was 19 days for those who withdrew, showing that the latter group stayed in the hospital statistically significantly longer $(p<0.01$, data not shown). In a single-arm pilot study, Schmidt et al. (20) found that participant motivation to eat meals influenced the induction of ketosis and the benefits received from meals. These results suggest that nutrition support team and medical professionals should actively intervene to motivate each patient to maximize the effects of the KD.

The KD group showed significantly higher overall subjective satisfaction with their meals. The greatest difference in satisfaction between the two groups was with regard to the volume of food, because, as previously mentioned, the GD meals were almost 3.5 times larger than the KD meals. In addition, contrary to the aforementioned view of poor fat digestion, our results about meal intake-related problems showed that KD did not add extra burden to the digestive system in post-pancreatectomy patients. These results suggest that KD is a safe way to increase energy and nutrient intake in pancreatobiliary cancer patients after surgery. 
The differences in meal compliance and nutrient intake rates between the KD group and GD group also induced differences in patients' nutritional status, biochemical parameters, and body compositions, though most of these differences were not statistically significant.

Nutritional status was assessed using PG-SGA grades. The proportion of patients in poor nutritional status (SGA B, SGA C) increased at DC in both groups, but the rate of increase was not statistically different. These results may have been due to the fact that both KD and GD groups were regularly assessed by a dietitian and were given nutritional interventions during the study.

This study's results showed that both groups had reduced serum lipid concentrations after surgery. This result was likely due to the reduction of blood cholesterol levels that occurs after physical traumas, as opposed to the food that the patients received. Numerous researchers reported that blood cholesterol concentration decreased substantially following major surgeries and that this reduced concentration persisted for approximately two weeks. The triglyceride concentration also decreases after surgery and returns to normal levels within several days (21-22). In this study, the lipid levels dropped in both groups and the level of decrease was not statistically different among groups. However, the reduction in LDL cholesterol levels tended to be greater in the KD group than in the GD group. These results were in line with the findings of Schmidt et al. (20) who found that providing protein and fat supplements in addition to carbohydraterestricted diet to cancer patients led to a reduction in total cholesterol and LDL-cholesterol levels.

Recent studies have reported that low-carbohydrate, highfat diets have benefits for weight loss and cardiovascular diseases by having a positive impact on lipid metabolism. Sharman et al. found that those who ate a diet consisting of $30 \%$ protein, $8 \%$ carbohydrates, and $61 \%$ fat had lower serum triglyceride concentration, high HDL-cholesterol concentration, and smaller LDL-cholesterol particles than those who ate a regular diet, which ultimately reduced cardiovascular risk (23). However, these studies were conducted on healthy adults, so additional studies are needed to investigate the effects of these types of diets on lipid metabolism in cancer patients. In this study, nuts and vegetable oils were used as major sources of fat. These foods are rich in polyunsaturated fatty acid and phytosterols which are known to improve lipid profile $(24,25)$. Therefore, the reduction of total cholesterol and LDL-cholesterol after eating the KD was likely due to the rich unsaturated fatty acid and phytosterol content of the diet.

Regarding body composition changes during the study period, the GD group maintained or mildly increased BFM after surgery but the KD group had significantly reduced BFM one month after surgery. Similar findings were reported in the study by Klement and Sweeney (26) in which a KD was provided to patients undergoing adjuvant radiotherapy. This study's findings were also in line with a study that found that a KD is effective in treating obesity (27). In this study, BCM and SMM decreased over time, but the degree of these changes was greater in the GD group than in the KD group $(p<0.05)$. The difference between GD and KD group was not significant after adjustment with age, but this finding suggests that ketogenic diet is beneficial in preserving lean body mass. A recent study reported that reduction of lean body mass and body weight is a sign of malnutrition (28). Therefore, this study showed that KD developed in this study is effective in preserving lean body mass and nutritional status of cancer patients after pancreatectomy.

In this study, urine ketones were detected more frequently in the KD, though the difference was not statistically significant. The KD group also had a significantly higher serum ketone body concentration ( $\beta$-hydroxybutyrate) than the GD group (unpublished data). So, it is clear that KD in this study has elevated ketone bodies concentration in the system. However, it should be addressed that there are a few differences between this study and previous studies administering a KD. First, the ketogenic ratio in this study was 1.05-1.75:1, which was different from the 4:1-3:1 ratio commonly reported in the literature. Furthermore, a previous study argued that a KD should be provided for at least three weeks in order to induce ketosis (11), but this study found an increase in urine ketone bodies within an average of two weeks or less. Moreover, ketone body analysis was qualitative, not quantitative in this study. Therefore, further studies are needed to clarify the dose and duration of this KD to induce ketosis. Also, more studies are necessary to elucidate the effect of $\mathrm{KD}$ in cancer metabolism of pancreatobiliary patients.

In summary, the KD designed in this study improved meal compliance, satisfaction, and energy intake rate in postpancreatectomy patients without increasing complications of the digestive system. Furthermore, it induced changes in the metabolic state, indicating that it can improve clinical parameters as well. In addition, urine ketone bodies tended to increase after consuming KD. Therefore, this study showed that KD is a safe adjuvant nutritional intervention in pancreatobiliary cancer treatment and provided foundational data for subsequent studies investigating the anti-cancer effects of KDs.

However, one limitation was that strict randomization was not possible because the participants were patients who needed immediate cancer surgeries. Additional randomized controlled trials conducted with a larger number of participants are needed to assess the effects of KD as an adjuvant anti-cancer therapy.

\section{Conflicts of Interest}

The Authors of this article have no conflicts of interest to declare. 


\section{Acknowledgements}

This work was supported by Korea Institute of Planning and Evaluation for Technology in Food, Agriculture, Forestry and Fisheries (iPET, 316055-3).

\section{References}

1 Ferlay J, Soerjomataram I, Dikshit R, Eser S, Mathers C, Rebelo M, Parkin DM, Forman D and Bray F: Cancer incidence and mortality worldwide: sources, methods and major patterns in GLOBOCAN 2012. Int J Cancer 136(5): E359-386, 2015.

2 Center NCI. Cancer Statistics. http://www.cancer.go.kr/lay1/ S1T639C641/contents.do.

3 Hartwig W, Hackert T, Hinz U, Gluth A, Bergmann F, Strobel $\mathrm{O}$, Buchler MW and Werner J: Pancreatic cancer surgery in the new millennium: better prediction of outcome. Ann Surg 254(2): 311-319, 2011.

4 Berry AJ: Pancreatic surgery: indications, complications, and implications for nutrition intervention. Nutr Clin Pract 28(3): 330-357, 2013.

5 Gilliland TM, Villafane-Ferriol N, Shah KP, Shah RM, Tran Cao HS, Massarweh NN, Silberfein EJ, Choi EA, Hsu C, McElhany AL, Barakat O, Fisher $\mathrm{W}$ and Van Buren G: Nutritional and metabolic derangements in pancreatic cancer and pancreatic resection. Nutrients 9(3): pii: E243, 2017.

6 Park JW, Jang JY, Kim EJ, Kang MJ, Kwon W, Chang YR, Han IW and Kim SW: Effects of pancreatectomy on nutritional state, pancreatic function and quality of life. Br J Surg 100(8): 10641070, 2013.

7 Pappas S, Krzywda E and McDowell N: Nutrition and pancreaticoduodenectomy. Nutr Clin Pract 25(3): 234-243, 2010.

8 Bozzetti F, Mariani L, Lo Vullo S, Group SW, Amerio ML, Biffi R, Caccialanza G, Capuano G, Correja I, Cozzaglio L, Di Leo A, Di Cosmo L, Finocchiaro C, Gavazzi C, Giannoni A, Magnanini P, Mantovani G, Pellegrini M, Rovera L, Sandri G, Tinivella $\mathrm{M}$ and Vigevani E: The nutritional risk in oncology: a study of 1,453 cancer outpatients. Support Care Cancer 20(8): 1919-1928, 2012

9 Kang J, Park JS, Yoon DS, Kim WJ, Chung HY, Lee SM and Chang N: A study on the dietary intake and the nutritional status among the pancreatic cancer surgical patients. Clin Nutr Res 5(4): 279-289, 2016.

10 Allen BG, Bhatia SK, Anderson CM, Eichenberger-Gilmore JM, Sibenaller ZA, Mapuskar KA, Schoenfeld JD, Buatti JM, Spitz DR and Fath MA: Ketogenic diets as an adjuvant cancer therapy: History and potential mechanism. Redox Biol 2: $963-$ 970, 2014

11 Oliveira CLP, Mattingly S, Schirrmacher R, Sawyer MB, Fine EJ and Prado CM: A Nutritional perspective of ketogenic diet in cancer: a narrative review. J Acad Nutr Diet 118(4): 668-688, 2018.

12 Weijs PJ, Kruizenga HM, van Dijk AE, van der Meij BS, Langius JA, Knol DL, Strack van Schijndel RJ and van Bokhorst-de van der Schueren MA: Validation of predictive equations for resting energy expenditure in adult outpatients and inpatients. Clin Nutr 27(1): 150-157, 2008.

13 Bauer J, Capra S and Ferguson M: Use of the scored PatientGenerated Subjective Global Assessment (PG-SGA) as a nutrition assessment tool in patients with cancer. Eur J Clin Nutr 56(8): 779, 2002.
14 Dindo D, Demartines N and Clavien P-A: Classification of surgical complications: a new proposal with evaluation in a cohort of 6336 patients and results of a survey. Ann Surg 240(2): 205, 2004.

15 Westman EC, Feinman RD, Mavropoulos JC, Vernon MC, Volek JS, Wortman JA, Yancy WS and Phinney SD: Low-carbohydrate nutrition and metabolism. Am J Clin Nutr 86(2): 276-284, 2007.

16 Schiesser M, Müller S, Kirchhoff P, Breitenstein S, Schäfer M and Clavien PA: Assessment of a novel screening score for nutritional risk in predicting complications in gastro-intestinal surgery. Clin Nutrit 27(4): 565-570, 2008.

17 Wallengren O, Lundholm K and Bosaeus I: Diet energy density and energy intake in palliative care cancer patients. Clin Nutr 24(2): 266-273, 2005.

18 De Waele E, Mattens S, Honore PM, Spapen H, De Greve J and Pen JJ: Nutrition therapy in cachectic cancer patients. The Tight Caloric Control (TiCaCo) pilot trial. Appetite 91: 298-301, 2015.

19 Langius JA, Zandbergen MC, Eerenstein SE, van Tulder MW, Leemans CR, Kramer MH and Weijs PJ: Effect of nutritional interventions on nutritional status, quality of life and mortality in patients with head and neck cancer receiving (chemo) radiotherapy: a systematic review. Clin Nutr 32(5): 671-678, 2013.

20 Schmidt M, Pfetzer N, Schwab M, Strauss I and Kämmerer U: Effects of a ketogenic diet on the quality of life in 16 patients with advanced cancer: A pilot trial. Nutr Metabol 8(1): 1-13, 2011.

21 Kesteloot H, Cobbaert C, Meyns B, Szecsi J, Lesaffre E and Sergeant P: Time course of serum lipid and lipoprotein levels after coronary bypass surgery: modification by pravastatin. Acta Cardiol 47(6): 519-528, 1992.

22 Malmendier CL, Amerijckx JP, Bihain BE and Fischer ML: Changes in apolipoprotein and lipids in patients after surgery. Biomed Pharmacother 39(4): 192-195, 1985.

23 Sharman MJ, Kraemer WJ, Love DM, Avery NG, Gómez AL, Scheett TP and Volek JS: A ketogenic diet favorably affects serum biomarkers for cardiovascular disease in normal-weight men. J Nutr 132(7): 1879-1885, 2002.

24 Kris-Etherton PM, Pearson TA, Wan Y, Hargrove RL, Moriarty $\mathrm{K}$, Fishell V and Etherton TD: High-monounsaturated fatty acid diets lower both plasma cholesterol and triacylglycerol concentrations. Am J Clin Nutr 70(6): 1009-1015, 1999.

25 Jones PJ, MacDougall DE, Ntanios F and Vanstone CA: Dietary phytosterols as cholesterol-lowering agents in humans. Can J Physiol Pharmacol 75(3): 217-227, 1997.

26 Klement RJ and Sweeney RA: Impact of a ketogenic diet intervention during radiotherapy on body composition: I. Initial clinical experience with six prospectively studied patients. BMC Res Notes 9(1): 143, 2016.

27 Paoli A: Ketogenic diet for obesity: friend or foe? Int J Environ Res Public Health 11(2): 2092-2107, 2014.

28 Jensen GL, Mirtallo J, Compher C, Dhaliwal R, Forbes A, Grijalba RF, Hardy G, Kondrup J, Labadarios D and Nyulasi I: Adult starvation and disease-related malnutrition: a proposal for etiology-based diagnosis in the clinical practice setting from the International Consensus Guideline Committee. J Parenter Enteral Nutr 34(2): 156-159, 2010.

Received September 18, 2018

Revised September 28, 2018 Accepted October 1, 2018 